\title{
DISCRETE SUFFICIENT SETS FOR SOME SPACES OF ENTIRE FUNCTIONS
}

\author{
BY \\ B. A. TAYLOR( $\left.{ }^{1}\right)$
}

\begin{abstract}
Let $E$ denote the space of all entire functions $f$ of exponential type (i.e. $|f(z)|=O(\exp (B|z|))$ for some $B>0)$. Let $\mathscr{K}$ denote the space of all positive continuous functions $k$ on the complex plane $C$ with $\exp (B|z|)=O(k(z))$ for each $B>0$. For $k \in \mathscr{K}$ and $S \subset C$, let $\|f\|_{k, S}=\sup \{|f(z)| / k(z): z \in S\}$. We prove that the two families of seminorms $\left\{\|\|_{k, C}\right\}_{k \in \mathscr{K}}$ and $\left\{\|\|_{k, s}\right\}_{k \in \mathscr{K}}$, where
\end{abstract}

$$
S=\{n+i m:-\infty<n, m<+\infty\},
$$

determine the same topology on $E$.

1. Introduction. Let $E=\{f$ entire $:|f(z)| \leqq A \exp (B|z|)$ for some $A, B>0\}$ denote the vector space of entire functions of exponential type, and let $\mathscr{K}$ denote the space of all positive continuous functions $k(z)$ on the complex plane $C$ such that $\exp (A|z|)=O(k(z))$ as $|z| \rightarrow \infty$ for each $A>0$. For each subset $S$ of $C, f \in E$, and each $k \in \mathscr{K}$, define

$$
\|f\|_{k, S}=\sup \{|f(z)| / k(z): z \in S\} .
$$

When $S=C$, write $\|f\|_{k}$ for $\|f\|_{k, C}$. The seminorms \|\|$_{k}$ determine a locally convex topology on $E$ which has been shown by L. Ehrenpreis [3, Chapter 5] (see also [10]) to be of interest in the study of several problems concerning entire functions. He has also posed the following question [3, p. 173]. Call a subset $S$ of $\boldsymbol{C}$ sufficient if the seminorms \|\|$_{k, s}$ determine the same topology on $E$ as do the seminorms \|\|$_{k}$. Are the lattice points $\{n+i m: n, m=0, \pm 1, \pm 2, \ldots\}$ a sufficient set? We prove here that this is the case.

The above problem is one of estimating entire functions which are "not too large" from their values at the lattice points, and such problems have been discussed by several authors (see e.g. [2], [5], [7], [8], [11], [12], [13], [14]). Work along these lines seems to have started from a problem posed by Littlewood and solved by G. Pólya, J. M. Whittaker, and others (see [12]). Namely, if $f$ is an entire function which is bounded at the lattice ponts and if

$$
M(r, f)=\sup \{|f(z)|:|z|=r\}
$$

Received by the editors February 8, 1971.

AMS 1970 subject classifications. Primary 46E10, 30A98; Secondary 30A64, 30A62. function.

Key words and phrases. Entire function, sufficient set, Fourier transform, subharmonic

(1) The author wishes to thank the National Science Foundation for support.

Copyright (C) 1972, American Mathematical Society 
satisfies $\log M(r, f)=o\left(r^{2}\right)$, then $f$ must be a constant. In connection with this problem, Whittaker has discussed the notion of "flat regions" of entire functions [14] (see also [7] and [8]). The work presented here was motivated by that of Whittaker and we have, in fact, given uniform versions of estimates similar to those proved by him (e.g. compare Lemma 3 with Lemma 504 of [14]). Other results related to the ones proved here are due to V. Ganapathy Iyer [5], whose notion of an "effective" set is about the same as a sufficient set.

One reason for determining "small" sufficient sets is that a set $S$ is sufficient if and only if every entire function $F(z)$ can be represented as an absolutely convergent Fourier integral

$$
F(z)=\int e^{i z w} d v(w)
$$

where the measure $v$ is supported on the set $S[3, \mathrm{p} .12$, or 4$]$. Thus, we obtain as a corollary the following fact.

COROLlaRY. Every entire function $F$ can be represented in the form

$$
F(z)=\sum_{n, m=-\infty}^{+\infty} a_{n, m} e^{(n+i m) z}
$$

where $\left|a_{n, m}\right|^{\left(n^{2}+m^{2}\right)^{-1 / 2}} \rightarrow 0$ as $n^{2}+m^{2} \rightarrow+\infty$.

The expansion for $F$ is never unique.

2. If $S$ is a subset of $C$, denote by $\rho(z, S)$ the distance from $z \in C$ to $S$.

THEOREM 1. If $\rho(z, S) \leqq 1$ for all $z \in C$, then $S$ is a sufficient set.

Theorem 1 will be deduced from the following estimate for entire functions and the lemma following it. For an entire function $f$, let

$$
M(r, f)=\sup \{|f(z)|:|z|=r\}
$$

be the maximum modulus function of $f$ and let

$$
M(r, f ; S)=\sup \{|f(z)|:|z| \leqq r, z \in S\} .
$$

THEOREM 2. Let $S \subset C$ be such that $\rho(z, S) \leqq 1$ for all $z \in C$, and let $f$ be an entire function with $f(0)=1$. There are absolute constants $C_{1}, C_{2}, C_{3}>0$ such that, for all $r \geqq 8$,

$$
\log M(r, f) \leqq \log ^{+} M(2 r, f ; S)+C_{1}\left\{\frac{\log M\left(C_{2} r\right)}{r}+\left(\frac{\log M\left(C_{2} r\right)}{r}\right)^{2}\right\}+C_{3} r .
$$

Lemma 1. Let $u(r), \varphi(r)$ be positive, increasing, continuous functions defined for $r \geqq 8$ and such that for some positive constants $C_{1}, C_{2}, C_{3}, K \geqq 1$

(i) $\sup _{r}\left(\varphi\left(C_{2} r\right) / \varphi(r)\right) \leqq K$,

(ii) $r=o(\varphi(r))$,

(iii) $\varphi(r)=o\left(r^{2}\right)$, 
(iv) $u(r)=o(\varphi(r))$,

(v) $u(r) \leqq \varphi(r)+C_{3} r+C_{1}\left\{u\left(C_{2} r\right) / r+\left(u\left(C_{2} r\right) / r\right)^{2}\right\}$.

Then there is a number $r_{0}>0$, depending only on $\varphi, C_{1}, C_{2}, C_{3}, K($ and not on $u(r)$ ) such that, for all $r \geqq r_{0}, u(r) \leqq 2\left\{\varphi(r)+C_{3} r\right\}$.

We will first deduce Theorem 1 from Theorem 2 and Lemma 1.

Proof of Theorem 1. Clearly the topology on $E$ induced by the seminorms \|\|$_{k, S}$ is weaker than that induced by the seminorms \|\|$_{k}$. Thus, we have to show that given any $k \in \mathscr{K}$, there exists $k_{1} \in \mathscr{K}$ and $C>0$ such that

$$
\|f\|_{k} \leqq C\|f\|_{k_{1}, s} .
$$

Actually, we are going to prove an estimate of the form

$$
\|f\|_{k} \leqq C\left(\|f\|_{k_{1}, \mathrm{~s}}\right)^{2}+D
$$

where $C, D$ are constants, but (1) follows from this by applying (2) to the function $\lambda f$ and then setting $\lambda=\|f\|_{k} / 2 C\left(\|f\|_{k_{1}, S}\right)^{2}$.

Let $k \in \mathscr{K}$. It is no loss of generality to assume that $0 \in S$, that $k(0)=1$, and that $k(z)=\exp \left(\varphi(|z|)+2 C_{3}|z|\right)$ where $\varphi$ is an increasing function satisfying (i)-(iii) of Lemma 1 and $C_{3}$ is the constant appearing in Theorem 2. Then set

$$
k_{1}(z)=\exp \left(\frac{1}{2} \varphi(|z| / 2)\right) \text {. }
$$

Now if $f(0)=1$ and $\|f\|_{k_{1}, S} \leqq A$ we have by Theorem 2 that

$$
\begin{aligned}
\log M(r, f) \leqq & \frac{1}{2} \varphi(r)+C_{3} r+\log A \\
& +C_{1}\left\{\frac{\log M\left(C_{2} r, f\right)}{r}+\left(\frac{\log M\left(C_{2} r, f\right)}{r}\right)^{2}\right\}
\end{aligned}
$$

Since $k_{1}(0)=k(0)=1$ we have

$$
1=\left|f(0) / k_{1}(0)\right| \leqq\|f\|_{k_{1}, s} \leqq A
$$

so we may apply Lemma 1 to deduce that there exists $r_{0}>0$, independent of $f$ and $A \geqq 1$, such that $\log M(r, f) \leqq \varphi(r)+2 C_{3} r+2 \log A$ or $\left|f\left(r e^{i \theta}\right)\right| \leqq A^{2} k\left(r e^{i \theta}\right)$ for $r \geqq r_{0}$. If $r \leqq r_{0}$ and if $C=\max \left\{k\left(r_{0}\right) / k(r): 0 \leqq r \leqq r_{0}\right\}$, then we have $\|f\|_{k} \leqq C A^{2}$. That is, if $f(0)=1$, then

$$
\|f\|_{k} \leqq C\left(\|f\|_{k_{1}, S}\right)^{2} .
$$

If $|f(0)| \geqq 1$, then we may apply (3) to $f \mid f(0)$ to deduce that the same inequality holds. If $|f(0)| \leqq 1$, then

$$
\begin{aligned}
\|f\|_{k} & =\|f-(f(0)-1)+(f(0)-1)\|_{k} \\
& \leqq\|f-(f(0)-1)\|_{k}+\|f(0)-1\|_{k} \\
& \leqq C\left(\|f-(f(0)-1)\|_{k_{1}, s}\right)^{2}+\sup \{2 / k(z): z \in C\} .
\end{aligned}
$$

Now $\|f-(f(0)-1)\|_{k_{1}, S} \leqq\|f\|_{k_{1}, S}+\|f(0)-1\|_{k_{1}, S}$ and $(a+b)^{2} \leqq 2\left(a^{2}+b^{2}\right)$ so when, $|f(0)| \leqq 1,\|f\|_{k} \leqq 2 C\left(\|f\|_{k_{1}, S}\right)^{2}+D$ where $D=\sup \{2 / k(z)\}+\sup \left(8 C /\left(k_{1}(z)\right)^{2}\right)$ and 
the supremums are over $z \in C$. Taken together with (3), this proves (2), which, completes the proof of Theorem 1 .

It remains to prove Lemma 1 and Theorem 2. We first prove the lemma.

Proof of Lemma 1. Let $\psi(r)=2\left\{\varphi(r)+C_{3} r\right\}$. Since $r=o(\varphi(r))$ and $\varphi(r)=o\left(r^{2}\right)$, there exists $r_{0}>0$ such that, for all $r \geqq r_{0}, \varphi(r) \geqq r$ and

$$
r^{2} \geqq 8 C_{1}\left(\varphi\left(C_{2} r\right)+C_{3} C_{2} r\right) \cdot \max \left(K, C_{2}\right)
$$

where

$$
K=\sup \left\{\varphi\left(C_{2} r\right) / \varphi(r): r \geqq 0\right\} .
$$

Suppose by way of contradiction that $u(r) \geqq \psi(r)$ for some $r \geqq r_{0}$. Then from (v) we have, since $u\left(C_{2} r\right) / r \geqq u(r) / r \geqq 1$,

$$
\varphi(r)+C_{3} r \leqq 2 C_{1}\left[u\left(C_{2} r\right) / r\right]^{2} .
$$

However, by (i),

$$
\begin{aligned}
\varphi(r)+C_{3} r & =\left[\varphi\left(C_{2} r\right) \varphi(r) / \varphi\left(C_{2} r\right)\right]+\left[C_{3} C_{2} r / C_{2}\right] \\
& \geqq\left[\max \left(K, C_{2}\right)\right]^{-1}\left(\varphi\left(C_{2} r\right)+C_{3} C_{2} r\right),
\end{aligned}
$$

so (5) together with (4) implies that $u\left(C_{2} r\right) \geqq \psi\left(C_{2} r\right)$. Repeating the above argument, we find that, for every $n=1,2, \ldots, u\left(C_{2}^{n} r\right) \geqq \psi\left(C_{2}^{n} r\right)$ which, together with (ii) and (iv), is a contradiction. Thus, $u(r) \leqq \psi(r)$ for all $r \geqq r_{0}$, as asserted.

To give the proof of Theorem 2 we introduce the following notation and prove three (easy) lemmas. Let the zeros of the entire function $f$ be denoted by $\left\{z_{j}\right\}$. Here we use the standard convention that each zero is repeated in the sequence $\left\{z_{j}\right\}$ as many times as its multiplicity as a zero of $f$. For each $\zeta \in C$ and $t>0$, let

$$
n(t, \zeta)=\sum_{|z,-\zeta| \leqq t} 1
$$

denote the number of $z_{j}$ in the disc $|z-\zeta| \leqq t$. Write $n(t)$ for $n(t, 0)$ and let $\lambda$ denote Lebesgue measure on $\boldsymbol{C}$.

Lemma 2. Let $R \geqq 4$. Then $n(2, \zeta)=0$ except possibly for a set of $\zeta$ in $|\zeta| \leqq R / 2$ of Lebesgue measure at most $4 \pi n(R)$.

Proof. Obvious.

The next lemma is essentially Lemma 504 of [14].

Lemma 3. Let $R>0$. Then for all $|\zeta| \leqq R / 2$ and every $\delta>0$

$$
n(t, \zeta) \leqq \frac{4 C}{\pi \delta} \frac{n(R, 0)}{R^{2}} t^{2}, \quad 1 \leqq|t| \leqq R / 2
$$

except possibly for $\zeta$ in a set of Lebesgue measure not exceeding $\pi \delta R^{2}$. Here $C$ is an absolute constant. 
Proof. For every $z_{j}$ with $\left|z_{j}\right| \leqq R$, let $h_{j}(\zeta)=1$ if $\left|\zeta-z_{j}\right| \leqq 1$ and $h_{j}(\zeta)=0$ otherwise. Then let $h=\sum h_{j},\left|z_{j}\right| \leqq R$. Obviously, $\|h\|_{1}=\int h d \lambda=\pi n(R)$. Furthermore, if we set

$$
v(t, \zeta)=\int_{|\zeta-z| \leqq t} h(z) d \lambda(z)
$$

then, for all $t \geqq 0, v(t, \zeta) \leqq \pi n(t+1, \zeta)$ and, for $|t| \leqq R-|\zeta|, \pi n(t, \zeta) \leqq v(t+1, \zeta)$.

Define $h^{*}(\zeta)=\sup \left\{v(t, \zeta)\left(\pi t^{2}\right)^{-1}: t>0\right\}$ to be the maximal function associated with $h$. By a theorem of Hardy and Littlewood (see e.g. [9]), there is a constant $C>0$ such that

$$
\lambda\left\{\zeta: h^{*}(\zeta)>\varepsilon\right\} \leqq(C / \varepsilon)\|h\|_{1}=(C \pi / \varepsilon) n(R, 0) .
$$

Choose $\varepsilon$ so that $(C / \varepsilon) n(R, 0)=\delta R^{2}$. Then

$$
n(t, \zeta) \leqq(\pi)^{-1} v(t+1, \zeta) \leqq \frac{C}{\delta} \frac{n(R, 0)}{R^{2}}(t+1)^{2}
$$

except for $\zeta$ in a set of measure not exceeding $\pi \delta R^{2}$. When $|\zeta| \leqq R / 2$ and $1 \leqq t \leqq R / 2$, (6) implies the inequality of the lemma.

For $x>0$, let $\log ^{+} x=\max (\log x, 0)$, and $\log ^{-} x=\log ^{+} x-\log x=-\min (\log x, 0)$.

LeMmA 4. Suppose $R \geqq 12$ and that $f$ is analytic for $|z| \leqq R$, with zeros $\left\{a_{n}\right\}\left(a_{n} \neq 0\right)$. Then for all complex numbers $z$ with $|z| \leqq 1$, we have either

$$
\log |f(z)| \leqq \frac{12}{R \pi} \int_{-\pi}^{\pi} \log ^{+}\left|f\left(R e^{i \theta}\right)\right| d \theta
$$

or else

$|\log | f(0)|-\log | f(z)||$

$$
\leqq \frac{12}{R \pi} \int_{-\pi}^{\pi} \log ^{+}\left|f\left(R e^{i \theta}\right)\right| d \theta+2 K \sum_{2 \leqq\left|a_{n}\right| \leqq R}\left|a_{n}\right|^{-1}+\left|\sum_{\left|a_{n}\right|<2} \log \right| \frac{R^{2}\left(z-a_{n}\right)}{a_{n}\left(R^{2}-\bar{a}_{n} z\right)}|| .
$$

Proof. We have by the Poisson-Jensen formula [6, p. 1] that $\log |f(z)|=u(z)+v(z)$ where

$$
u(z)=\sum \log \left|\frac{R\left(z-a_{n}\right)}{R^{2}-\bar{a}_{n} z}\right| \leqq 0
$$

and

$$
v(z)=\frac{1}{2 \pi} \int_{-\pi}^{\pi} P(R, \theta, z) \log \left|f\left(R e^{i \theta}\right)\right| d \theta
$$

where $P(R, \theta, z)=\left(R^{2}-|z|^{2}\right) /\left|R e^{i \theta}-z\right|^{2}$. With $\log ^{+}|f|=\max (\log |f|, 0)$ and $\log ^{-}|f|=-\min (\log |f|, 0)$, we have from (7) that

$$
\frac{1}{2 \pi} \int_{-\pi}^{\pi} \log { }^{-}\left|f\left(R e^{i \theta}\right)\right| d \theta=\frac{1}{2 \pi} \int_{-\pi}^{\pi} \log ^{+}\left|f\left(\operatorname{Re}^{i \theta}\right)\right| d \theta-v(0) .
$$


Now, for $|z| \leqq 1$ and $R \geqq 2$ we have

$$
|P(R, \theta, z)-P(R, \theta, 0)| \leqq 2|z|(R+|z|) /(R-|z|)^{2} \leqq 12 / R,
$$

so that

$$
\begin{aligned}
|v(0)-v(z)| & \leqq \frac{6}{R \pi} \int_{-\pi}^{\pi}|\log | f\left(R e^{i \theta}\right)|| d \theta \\
& \leqq \frac{12}{R}\left\{\frac{1}{\pi} \int_{-\pi}^{\pi} \log ^{+}\left|f\left(R e^{i \theta}\right)\right| d \theta-v(0)\right\}
\end{aligned}
$$

If $v(0) \leqq 0$, then for $R \geqq 12$ it follows that $v(z) \leqq(12 / R \pi) \int_{-\pi}^{\pi} \log ^{+}\left|f\left(R e^{i \theta}\right)\right| d \theta$ which implies the first estimate of the lemma because $\log |f(z)| \leqq v(z)$. Thus, we may assume $v(0) \geqq 0$ so that

$$
|v(0)-v(z)| \leqq \frac{12}{R \pi} \int_{-\pi}^{\pi} \log ^{+}\left|f\left(R e^{i \theta}\right)\right| d \theta .
$$

Next, we have

$$
\log \frac{|a|}{R}-\log \left|\frac{R(z-a)}{R^{2}-\bar{a} z}\right|=-\log \left|1-\frac{z}{a}\right|+\log \left|1-\frac{\bar{a} z}{R^{2}}\right|
$$

so if $K$ is any constant such that $|\log (1-\zeta)| \leqq K|\zeta|$ for $|\zeta| \leqq \frac{1}{2}$, then for $|z| \leqq 1$,

$$
|u(0)-u(z)| \leqq\left|\sum_{\left|a_{n}\right|<2} \log \right| \frac{R^{2}\left(z-a_{n}\right)}{a_{n}\left(R^{2}-\bar{a}_{n} z\right)}||+K \sum_{2 \leqq\left|a_{n}\right| \leqq R}\left(\frac{1}{\left|a_{n}\right|}+\frac{1}{R}\right) .
$$

Since $\left|a_{n}\right| \leqq R$, the last estimate of the lemma follows from (8) and (9).

Proof of Theorem 2. Let $a \in C$, with $3|a| / 2 \geqq 12$. Since $\log |f|$ is subharmonic, $\log |f(a)|$ does not exceed the average of $\log |f|$ over the disc

$$
D_{a}=\{z:|z-a| \leqq|a| / 2\} \text {. }
$$

Consequently, if $E$ is any subset of this disc, we have

$$
\begin{aligned}
\log |f(a)| & \leqq \frac{4}{\pi|a|^{2}}\left\{\int_{E}+\int_{D_{a} \mid E} \log |f| d \lambda\right\} \\
& \leqq \sup _{\zeta \in E}\{\log |f(\zeta)|\}+\frac{4 \lambda\left(D_{a} \mid E\right)}{\pi|a|^{2}} \log M\left(\frac{3}{2}|a|, f\right)
\end{aligned}
$$

where $D_{a} \backslash E$ is the complement of $E$ in $D_{a}$.

Now, choose $\delta=n(3|a|, 0) /|a|^{2}$ and let $E$ denote the set of points in $D_{a}$ such that $n(2, \zeta)=0$ and

$$
n(t, \zeta) \leqq \frac{4 C}{9 \pi \delta} \frac{n(3|a|, 0)}{|a|^{2}} t^{2}, \quad 1 \leqq t \leqq \frac{3|a|}{2},
$$

where $C$ is as in Lemma 3. If we apply Lemmas 2 and 3 with $R=3|a|$, we see that

$$
\lambda\left(D_{a} \mid E\right) \leqq 4 \pi n(3|a|, 0)+9 \pi \delta|a|^{2}=13 \pi n(3|a|, 0) .
$$

If $\zeta \in E$, then apply Lemma 4 to the function $z \rightarrow f(\zeta+z)$, with $R=3|a| / 2$ to obtain 


$$
\begin{aligned}
\log |f(\zeta)| \leqq & \sup \left\{\log ^{+}|f(z)|: z \in S,|z| \leqq 3|a| / 2+1\right\} \\
& +\frac{16}{|a|} \log M(3|a|, f)+2 K \sum_{2 \leqq\left|z_{n}-\zeta\right| \leqq R}\left|z_{n}-\zeta\right|^{-1} .
\end{aligned}
$$

However,

$$
\sum_{2 \leqq\left|z_{n}-\zeta\right| \leqq R} \frac{1}{\left|z_{n}-\zeta\right|}=\int_{2}^{R} t^{-1} d n(t, \zeta) \leqq \frac{n(R, \zeta)}{R}+\int_{2}^{R} \frac{n(t, \zeta)}{t^{2}} d t .
$$

By (11) the last integral does not exceed

$$
\frac{4 C}{\pi \delta} \frac{n(3|a|, 0)}{9|a|^{2}} \cdot \frac{3}{2}|a|=\frac{2}{3} \frac{C}{\pi}|a|
$$

so that

$$
\begin{aligned}
\log |f(\zeta)| \leqq & \log ^{+} M(2|a|, f, S)+(16 /|a|) \log M(3|a|, f) \\
& +K \frac{n(3|a|, 0)}{3|a|}+\frac{4}{3} \frac{C K}{\pi}|a|
\end{aligned}
$$

Since for all $r>0, n(r, 0) \leqq \log M(e r, f)-\log |f(0)|$, the theorem follows directly from (10), (12) and (13).

REMARK 1. Exactly the same argument shows that any set $S$ with $\rho(z, S) \leqq 1$ is sufficient for the analytically uniform spaces of [10] for which the weights $k$ can be taken in the form $k(z)=\exp (\varphi(|z|))$ where $\varphi(z)=o\left(|z|^{2}\right)$ and for which $k_{1}(z)$ $=k^{1 / 2}(2 z)$ belongs to $\mathscr{K}$ whenever $k \in \mathscr{K}$.

REMARK 2. The condition $\rho(z, S) \leqq 1$ in Theorems 1 and 2 may obviously be replaced by $\rho(z, S) \leqq d$ for $d$ any positive constant.

\section{REFERENCES}

1. R. P. Boas, Entire functions, Academic Press, New York, 1954. MR 16, 914.

2. M. L. Cartwright, On functions bounded at the lattice points in an angle, Proc. London Math. Soc. 43 (1937), 26-32.

3. L. Ehrenpreis, Fourier analysis in several complex variables, Interscience, New York, 1970.

4. - Analytically uniform spaces and some applications, Trans. Amer. Math. Soc. 101 (1961), 52-74. MR 24 \#A1604.

5. V. Ganapathy Iyer, On effective sets of points in relation to integral functions, Trans. Amer. Math. Soc. 42 (1937), 358-365; correction, ibid. 43 (1938), 494.

6. W. K. Hayman, Meromorphic functions, Oxford Math. Monographs, Clarendon Press, Oxford, 1964. MR 29 \#1337.

7. A. J. MacIntyre, Wiman's method and the "flat regions" of integral functions, Quart. J. Math. Oxford Ser. (2) 9 (1938), 81-88.

8. A. Pfluger, On analytic functions bounded at the lattice points, Proc. London Math. Soc. 42 (1936), 305-315.

9. Keith Phillips, The maximal theorems of Hardy and Littlewood, Amer. Math. Monthly 74 (1967), 648-660. MR 35 \#6788. 
10. B. A. Taylor, Some locally convex spaces of entire functions, Proc. Sympos. Pure Math., vol. 11, Amer. Math. Soc., Providence, R. I., 1968, pp. 431-467.

11. G. Valiron, Sur les variations du module des fonctions entières ou méromorphes, C. $\mathbf{R}$. Acad. Sci. Paris 204 (1937), 33-35.

12. J. M. Whittaker, On the "flat" regions of integral functions of finite order, Proc. Edinburgh Math. Soc. 2 (1930), 111-128.

13. - On the fluctuation of integral and meromorphic functions, Proc. London Math. Soc. 37 (1934), 383-401.

14. - Interpolatory function theory, Cambridge Tracts in Math., no. 33, Cambridge Univ. Press, New York, 1935.

Department of Mathematics, University of Michigan, Ann Arbor, Michigan 48104 